\title{
Energy Filtered Transmission Electron Microscopy (EFTEM) and the use of Image-Spectroscopy
}

\author{
P.A.Midgley
}

Department of Materials Science and Metallurgy, University of Cambridge, Pembroke Street, Cambridge, CB2 3QZ, UK

The increasing availability of both post-column energy filters, such as the Gatan Imaging Filter (GIF), and incolumn filters such as the $\Omega$-filter on JEOL and LEO instruments, has led to an increasing use of energyfiltered TEM (EFTEM) for the 2D compositional analysis of materials using elemental and jump-ratio maps. The advantage of EFTEM over energy-loss spectroscopy is one of speed of mapping, with a large area map obtained in just a few minutes. To achieve such rapid mapping, however, most spectral information is lost and routine analysis often produces artefacts through poor statistics and poor pre-edge background fitting.

'EFTEM spectrum-imaging' or 'image-spectroscopy' [1], as it perhaps more correctly named, develops EFTEM into a more quantitative tool without sacrificing greatly the speed of analysis. Unlike conventional EFTEM in which only 3 images are recorded, with image-spectroscopy a large series of images are acquired sometimes only over a few 10 's of eV, sometimes ranging from zero to $3000 \mathrm{eV}$. With the introduction of simple interface scripting this process can be made automatic and reliable - it is as easy to acquire 100 images as it is just 3 ! In order to record such a data set over a large energy loss it is important to take into account the need to re-bin the data on the CCD, to perhaps change the energy-selecting slit width and to adjust acquisition times accordingly. It is vital that the illumination conditions are kept constant and this can be pre-calibrated. With a typical series, it may be necessary to acquire a zero loss image for $0.1 \mathrm{~s}$ at $1 \times$ binning with $10 \mathrm{eV}$ width, but an image at $2500 \mathrm{eV}$ loss for $16 \mathrm{~s}$ with $8 \times$ binning and with a $50 \mathrm{eV}$ slit width. Such parameters can be prejudged and it is important that every energy-loss image has sufficient counts to achieve meaningful statistics. Finally all images must be aligned to account for drift (using cross-correlation algorithms or a patient operator!) and all images scaled to account for changes to the acquisition conditions.

The array of energy-loss images can now be thought of as a 2D array of energy loss 'image-spectra' with a resolution equal to the slit width, far worse than conventional EELS. Nevertheless even with reduced energy resolution it is still possible to apply standard EELS analysis, such as improved background fitting (because of the greatly increased number of data points, see Fig. 1) and Fourier-based deconvolution routines that account for variations in thickness and subsequent plural scattering [2]. Elemental maps can be calculated from the series easily and accurately. Furthermore it is possible to sum a number of post-edge images to improve the overall signal-to-noise ratio without loss of spatial resolution. For example, it is possible to add 10 images each $10 \mathrm{eV}$ apart to give as good a signal as a single image with $100 \mathrm{eV}$ slit width but with far better spatial resolution. The optimum number of images to sum can be calculated using a routine found in [3]. Fig. 2 shows a montage of core-loss elemental maps of a precipitate cluster acquired from a 316-type stainless steel. The maps were constructed from a series of 80 images and Fourier-log deconvoluted to remove plural scattering effects. An image-spectrum line trace illustrates the extended dynamic range obtained by varying image integration times and pixel binning. The titanium nitride 'core' was unexpected and would have almost certainly been missed using conventional EFTEM [4].

\section{References}

[1] P.J. Thomas and P.A.Midgley, Ultramicroscopy 88 (2001) 179; ibid. 88 (2001) 187.

[2] R.F. Egerton, Electron energy-loss spectroscopy in the electron microscope. Plenum Press, New York and London, 1996.

[3] G. Kothleitner and F. Hofer, Micron 29 (1998) 349.

[4] This research was funded by the EPSRC. 

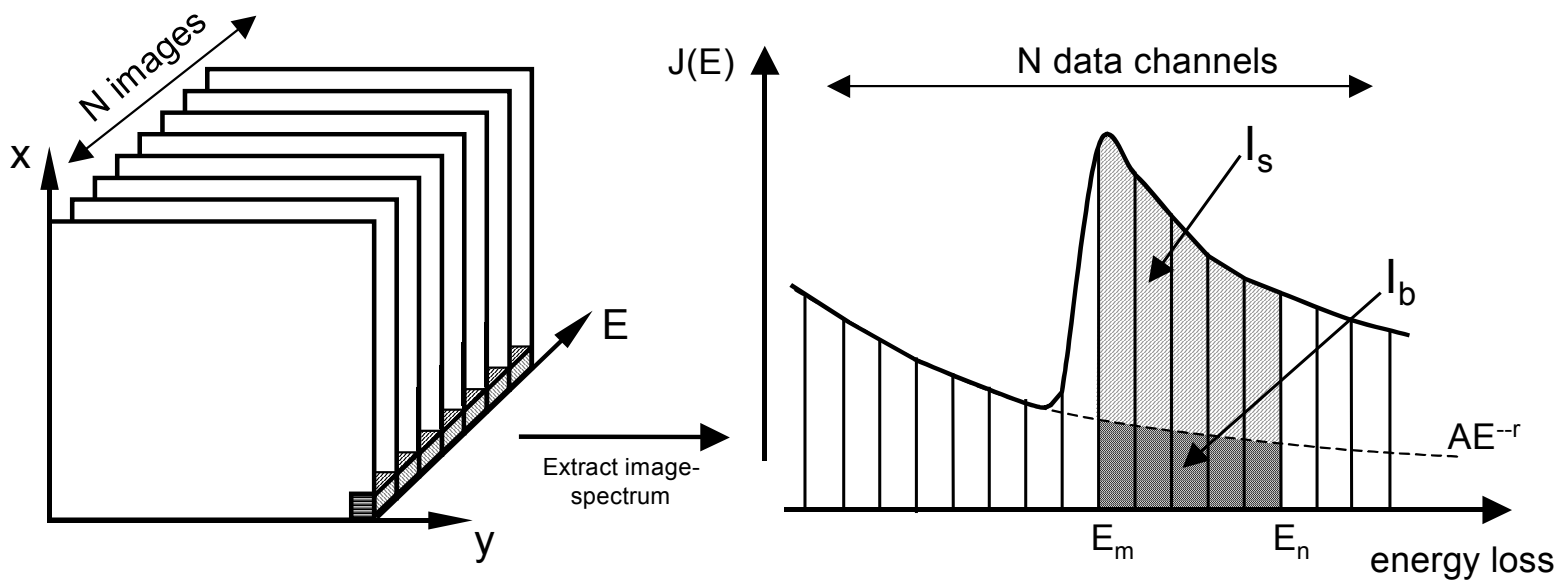

FIG. 1 Analysis of the core-loss signal using an extended image-series. $N$ images are acquired, allowing individual 'image-spectra' to be extracted, after correction for sample drift during acquisition, for analysis using conventional EELS analysis techniques.

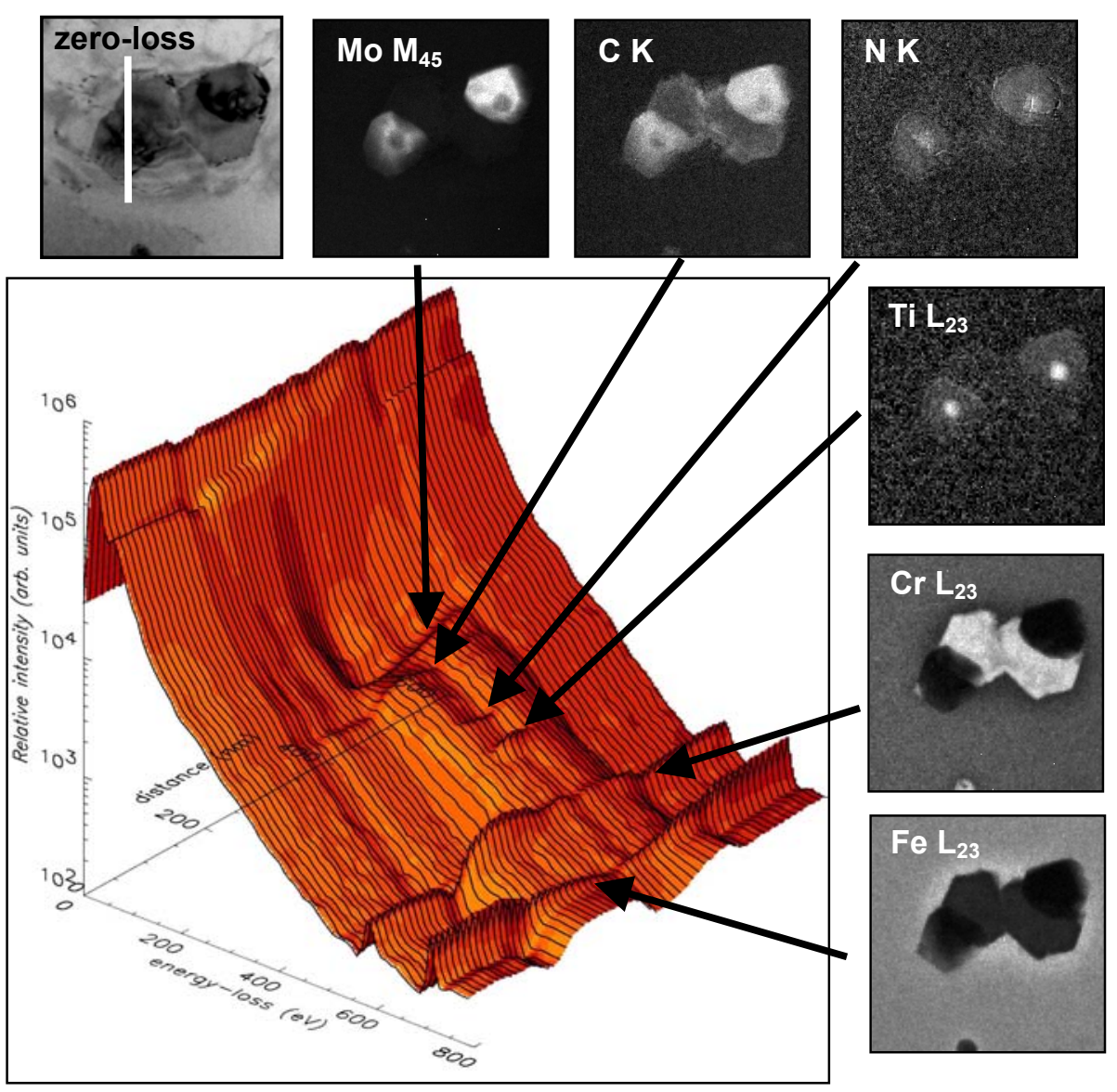

FIG.2 A montage of core-loss elemental maps and a zero loss image of a precipitate cluster. The maps were constructed from a series of 80 images acquired from a 316-type stainless steel. An image-spectrum line trace across the vertical line marked in the zero-loss image illustrates the extended dynamic range obtained by varying image integration times and pixel binning. A number of core-loss edges, acquired within the series, are revealed. 